\title{
Graduate EduCation in Physical OCEANOGRAPHY
}

\author{
By Joseph Pedlosky
}

$\mathrm{G}$ REAT WAVES of reform regularly sweep through every educational enterprise in science. The most natural explanation for this is a dissatisfaction most scientists feel about the education they themselves received as well as the education they are giving to their students. Physical oceanography has witnessed a quiet but radical transformation over the last quarter century in the manner in which new recruits to the field are educated.

It is now common for students to prepare themselves for careers in Physical Oceanography at institutions where they are instructed by teachers who themselves were trained as physical oceanographers. This contrasts with an older practice in which students, trained outside oceanography in basic physics or engineering, entered the field as full working scientists. In some especially striking cases, people entered essentially as apprentices with little or no training in either oceanography or any other basic science!

Although oceanography advanced remarkably far at the hands of such talented intellectual immigrants, this approach has been largely superseded by a more formal program of professional education. It seems like a good time to wonder about the way our program for education has developed and to critically examine where the next great wave should carry us. I am a scientist and a teacher and am writing from my experience in the MIT/Woods Hole Oceanographic Institution (WHOI) Joint Program rather than as an administrator with a broad statistical understanding of the national picture. However, I believe that the MIT/WHOI program is among the better programs, and I hope, therefore, that these reflections will have some general interest.

Physical oceanography is the study of the physics of the oceans, with a special focus on the dynamics of oceanic currents and waves. This physics is essentially nonlinear. The fluid dynam-

J. Pedlosky, Woods Hole Oceanographic Institution, Woods Hole, MA 02543, USA. ics at the heart of the subject naturally link phenomena of vastly different time and space scales. Currents like the Gulf Stream, eddies and gravity waves, small convective plumes, and "fingers" are all interacting elements of an immensely intricate whole. Important events occur within seconds whereas others are persistent enough to contribute to changes in the Earth's climate. The oceans stir the chemicals introduced both naturally and artificially by humans, and the moving waters are the habitat for a vigorous world of living beings. The oceans help shape and interact with the vessels that contain them and they drive and are driven by the Earth's atmosphere. How, in any reasonable time, are we to train new physical oceanographers to deal with such a vast subject?

\section{Goals in Education}

It seems to me that the first item of business is to agree on the proper goal of a graduate program in physical oceanography. This is not as easy as it sounds for there are at several conflicting attitudes commonly current in the field. The first and more traditional of these sees oceanography as a single unitary whole. All branches of oceanography, i.e., physical, chemical, biological and geological, are seen as closely fitting parts of a single science. The task of education in this traditional view is to make sure each student knows something about all branches of oceanography. This attitude is typified by courses that at least philosophically follow in the pattern of the great oceanographic treatise, The Oceans (Sverdrup et al., 1942). This massive text, which runs to over a thousand pages, imposes a suggestion of a basic curriculum in oceanography. It treats all the subjects described above and has had an enormous influence in our thinking about oceanographic education. It is a seductive suggestion because each of its parts is engaging intellectually and there are few who would say that they prefer not to know the content of each section. Breadth in education is considered a good thing, and if oceanography is thought of as a self-contained whole then this
. . a good time to

wonder about the way our program for education has developed . . . 


\section{$\mathrm{M}_{\mathrm{y} \text { argument is ... }}$ \\ whether the traditional \\ prescription of breadth \\ . . is the correct one.}

approach holds out a vision of the "broad" education. I think, though, that for physical oceanography there are serious problems with this attitude. It is recognized even by the authors of The Oceans, who remark that "In the field of physical oceanography, the greater part of the theoretical and practical work can be conducted with little or no attention to results in the other marine sciences."

In fact, I claim it is easy to argue that for physical oceanographers breadth in education demands preferentially the study of meteorology, mathematics, engineering, and advanced topics in other branches of physics. These are often the more natural intellectual sources of advancement for our science. My argument is therefore not with breadth versus depth but partly with whether the traditional prescription of breadth, as manifested in The Oceans, is the correct one.

Most of our students come to be educated to do research in physical oceanography. Our goal in the MIT/WHOI Joint Program is to enable them to become successful independent researchers. The test of whether we have succeeded is generally considered to be equivalent to asking whether the student has, at the end, written a thesis that is a creative stimulating piece of work that advances the field and is a genuine earnest of the student's advancement to the ranks of independent research scientists. From this second and less traditional point of view, it is what the student ends up being able to do rather than how much the student knows that is the best measure of the education program. Of course these are not antithetical goals in principle. However, our principal goal is the development of the creative scientist rather than the simply well-informed student.

How do we try to go about this process now? The transformation from an undergraduate receptor of information into a graduate student carrying out his or her own advanced research is still a rather mysterious one. What do we do now to make it work and what could we do better in the future?

\section{What Do We Teach?}

Most of the students entering our graduate program in physical oceanography have no academic experience with oceanography. They are attracted to the field by a variety of idiosyncratic reasons, and many have a very vague notion of what our field is all about. The ones that do best in the program, or at least the applicants we feel are most desirable, are those with strong backgrounds in physics and mathematics.

As physics is taught in most universities today, the new applicant is likely to be innocent of any knowledge of fluid dynamics, which is that branch of classical physics of most direct application in physical oceanography. Although fluid mechanics is a still rapidly evolving subject of the greatest beauty and challenge, it has long been considered to be an "applied" physics subject and has disappeared from most physics departments' curricula. The upsurge of interest in the chaotic behavior of nonlinear dynamical systems, which was made evident first in the fluid dynamics of the atmosphere, may change this circumstance. However, in the foreseeable future one of the main tasks of our educational effort is furnishing our students with the necessary background in fluid mechanics.

We have developed a 2-year program of courses in fluid dynamics. Starting with the basic formulation of fluid mechanics as a subject in continuum physics, the student is led through a sequence of four one-semester courses. The topics cover wave dynamics, the steady circulation physics of the oceans (and atmosphere), and the particular dynamics of the synoptic or eddy scale in the ocean. Additional theoretical courses deal with the dynamics peculiar to coastal regions, in which the influence of the relatively shallow continental shelf and slope are important. Advanced courses in stability theory, strong eddies, air-sea interaction, and modern ideas of the general circulation serve to supplement the offerings of the core program. The student is also encouraged to continue his or her training in applied mathematics by following courses taught in the MIT applied mathematics program. Simultaneously, the student is offered a set of courses dealing with the descriptive and experimental aspects of physical oceanography. It is important that students be aware of the principal physical characteristics and phenomena of the oceans and at the same time have an understanding for how experiments are planned, how observations are actually made, and how the data, once obtained, can be usefully analyzed and interpreted. Whether a student's interest tends to the experimental or the theoretical, the common unifying language is the dynamics of fluids. Thus, the central emphasis of our educational program is designed in the hope that the student's foundation in fluid mechanics is sturdy and fundamental enough to support the developments that will come in the field and the evolving research directions the student will follow later in his or her career.

This represents quite an intense program of instruction. Yet we expect that students will complete their formal course work during their first 2 years in the joint program and, after entering candidacy in their third year, will be ready to entirely immerse themselves in their dissertation research. Even a very well-organized program focused on the physics of the oceans has difficulty in providing complete exposure to all areas that would be ideal. Compromises are inevitable, and this leads to the 
need to re-examine critically the formal part of the educational program. Gaps are inescapable in any one student's training. I believe that each of us as working scientists is aware of our own deficiencies in a number of important areas of physical oceanography.

Although we each seem able to carry on our own research in spite of these gaps, there is a natural tendency to require that our students not share our own deficiencies. This laudable tendency, if carried to its natural end by an entire faculty, can lead to a saturation of formal information that is as paralyzing to creative research as the traditional "all branches of oceanography" program $\mathbf{I}$ described earlier. At the least, to encourage our students to get on with the important job of doing and learning how to do research, we should teach them to live with a certain incompleteness in their formal preparation.

\section{The Students}

Most of our students come from an undergraduate background in science as taught in the American liberal-arts tradition. A smaller number come from technical universities such as MIT. In common with other branches of science, we have found that the pool of acceptable applicants to the oceanography graduate program has shrunk, although the applicant pool in physical oceanography has hovered at the same number for about a decade. Typically, $\sim 25$ students apply to us for admission in physical oceanography; we accept $\sim 10$ and 6 or 7 decide to enter. These are small numbers, and it is hard to feel confident about making any general statement about the students, except that the number of applicants is uncomfortably small to ensure for the future the stream of high-quality researchers the field will need.

A continuing concern among the faculty is the perceived decline in the mathematical backgrounds of the admitted students, apart from those foreign students whose mathematical preparation is frequently very strong. Too many students in the first years are struggling over the mathematical aspects of their courses and missing out on the physical content that the mathematics is supposed to illuminate. In the MIT/WHOI Joint Program for Physical Oceanography, we have at any one time between 25 and 30 students who take anywhere from $4 \frac{1}{2}$ to $6 \frac{1}{2}$ years to finish their PhDs, although $5 \frac{1}{2}$ years is most typical. They work on their research in close consultation with the advisors they have at both MIT and Woods Hole. About $55 \%$ of the entering class of students will leave with their doctoral degrees, the rest will typically leave with a Masters degree, and a small number who have quickly discovered they are in the wrong program will leave with no degree at all. The successful graduates go almost without exception into an academic or government research career. A typical first position is a postdoctoral appointment at another oceanography department.

The thesis topics of our students range widely over both theoretical and experimental oceanographic physics. Some recent research projects have been observational studies of the equatorial current systems, combined modeling and description for entrainment of tracers into the Gulf Stream system, the buoyancy and wind-driven general circulation, and the instability of strong oceanic currents. These give some feeling for the wide variety of problems in physics that our students have successfully come to grips with during their thesis research.

\section{Where Do We Go Now?}

Our graduates populate the faculties of many universities and contribute to the current progress in physical oceanography. This success parallels the success of similar education programs at our sister institutions. Nevertheless, there are questions that are worth thinking about and that deal with concerns of common interest. Three are particularly interesting to me. They relate to 1) the issue of narrowness of education, 2) whether our mode of teaching students to do research can be improved, and 3) whether our national resources in physical oceanography are being used optimally.

1) Our students are usually very conscientious and ambitious. They are eager to become professional oceanographers very quickly. This is particularly poignant because many of us are not completely sure ourselves how to define what a physical oceanographer should know now or will need to know in the future (aside from some basics in fluid dynamics and mathematics). Yet, as I described earlier, the number of physical oceanography courses keeps growing as we try to close those terrifying gaps in preparation. It has become increasingly difficult to persuade students to take courses outside oceanography in collateral fields whose application to oceanography is not immediately apparent. My concern here is whether we are producing a breed of overspecialized professionals.

To some extent graduate education regardless of field should be a liberating and enriching experience rather than a narrowing one. Forecasts of the next 20 years in academia stress the need to replace a large number of retiring faculty in many scientific disciplines. Vacancies in physics and mathematics departments will cause those departments to rethink the character of the research that goes on within them. Will our graduates in oceanographic physics have a broad enough perspective on science to present themselves as candidates for those positions? If the an-
$T$ oo many students . . . are struggling over the mathematical aspects of their courses and missing out on the physical content . . . 
. . a student could

be admitted on a

national competitive

basis to a program run

by a consortium of

schools. swer is yes then that says something healthy and positive about the education we offer. If we have strong doubts about the answer, then perhaps it is time to think about the requirements of our program. It might be a good idea for us to accept more gaps in the professional training of oceanographers in exchange for a curriculum that encourages the student to experiment with courses in other branches of science with no apparent immediate utility in oceanography.

2) One of the big hurdles our students face is actually getting down to do research. The formal course work is psychologically a continuation of their undergraduate studies. We are so concerned that students have sufficient background in physical oceanography that really serious research often starts after the first 2 years have passed. I want to stress the difference between this dissertation research and the research project of the student's earlier years, which is usually modest in scope and rarely presents the student with the agony and frustration of taking on a big problem "in the dark." It is at this stage that most students have their biggest difficulties and face the sharpest need to reappraise their career goals. Should it occur so late? Are we using the courses as impediments to developing skills as researchers, which is our overall goal for our educational program? Perhaps, as in an experiment at Harvard Medical School, we should abandon completely the classical lecture or demonstration format for passing on information. Instead one could envision an educational environment in which groups of students are presented with carefully selected problems, not necessarily with known answers, and asked to learn what is needed to make progress toward solving the problems. Or they may be asked to learn, with the appropriate oversight and guidance, how to reformulate and redirect the problem's direction. This might give a more realistic training in what research in oceanic physics is all about. Initially, the greatest burden would be on the faculty. One of the appeals of the present lecture course is that after the first year, it places a much lower burden on the faculty member than close supervision of a more unpredictable research effort. I think alternatives like this should be considered on an experimental basis in our oceanographic programs. Otherwise, our programs will become increasingly unwieldy as the accumulation of new information about the oceans accelerates.

3) The number of students that enter physical oceanography each year is small. There are $\sim 60$ 70 successful applicants to all schools nationwide.
For those of us who take part in the admissions process, the shrinking number of acceptable applicants in the pool has led to a kind of frenzied competition between schools for the best of the applicants. The field of physical oceanography is still small enough to wonder about an alternative approach, in which the MIT/WHOI collaboration might serve as a model. MIT and WHOI are two independent degree-granting institutions. In principle, they too could provide independent programs in oceanography and compete for the same students. Instead, this competition, to the benefit of both the two institutions and the students, is substituted by a cooperative program in which the students can take advantage of the resources of both institutions. Bureaucratic problems dealing with the administration of the program have been handled well, primarily because everyone involved senses the immense advantages of the cooperative effort. Intrinsic problems dealing with the physical separation of the two campuses have been dealt with in a variety of imaginative ways, including the use of a two-way video link for classes and meetings.

With the enhanced communications technology that the next decades will surely bring, we should ask ourselves whether even broader cooperative arrangements between schools is not possible. I can envision very extensive arrangements in which a student could be admitted on a national competitive basis to a program run by a consortium of schools. The student could take advantage of teaching and research guidance offered by the whole array of institutions. Flexible cross-registration and interinstitutional dissertation guidance would yield benefits both to the students and to schools unable to provide a full range of teaching and research resources. Problems of tuition credit and satisfaction of degree requirements or physical commuting are no different in kind than those already solved in the cooperative MIT/WHOI program. Instead of competing for the top students, the consortium would represent a potent recruiting tool for physical oceanography within science as a whole. I believe it is not a moment too soon to start discussions of the radically different form graduate education in physical oceanography could effectively take in the twentyfirst century.

\section{References}

Sverdrup, H.U., M.W. Johnson and R.W. Fleming, 1942: The Oceans: Their Physics, Chemistry, and General Biology. Prentice-Hall, Englewood Cliffs, NJ, 1060 pp. $\square$ 